\title{
Experimentation on the Use of Chromaticity Features, Local Binary Pattern, and Discrete Cosine Transform in Colour Texture Analysis
}

\author{
Padmapriya Nammalwar, Ovidiu Ghita, and Paul F. Whelan \\ Vision Systems Group, School of Electronic Engineering, \\ Dublin City University, Dublin 9, Ireland \\ \{padmapri, ghitao, paul.whelan\}@eeng.dcu.ie
}

\begin{abstract}
This paper describes a method for colour texture analysis, which performs segmentation based on colour and texture information. The main goal of this approach is to examine the contribution of chromaticity features in the analysis of texture. Local Binary Pattern and Discrete Cosine Transform are the techniques utilised as a tool to perform feature extraction. Segmentation is carried out based on an unsupervised texture segmentation method. The performance of the method is evaluated using different chromaticity features and also using the ROC curves. The results indicate that the inclusion of colour information improves the segmentation performance.
\end{abstract}

\section{Introduction}

Colour and texture are the two naturally related characteristics of the image, but these features are often analysed separately. In the past few years, several studies have been directed to the problem of joint representation of texture and colour. Most textures have a colour aspect and most colour surfaces are textured. A colour texture can be regarded as a pattern described by the relationship between its chromatic and structural distribution [1. Segmentation of colour texture images involves identifying the boundary maps between various uniform regions. There are numerous techniques for the extraction of texture features and various methods for segmentation. Most of the methods are developed for greyscale textures, rather than for colour images. Recent studies have begun to show radiance on colour texture analysis.

Panjwani and Healey [2] presented an unsupervised segmentation algorithm based on Markov Random Field models for colour textures. Their models characterises a texture in terms of spatial interaction within each colour plane and interaction between different colour planes. Jain and Healey [3] introduced a method based on unichrome features computed from the three spectral bands independently and opponent features that utilise the spatial correlation between spectral bands using Gabor filters. Paschos [4] presented a visual monitoring system which incorporates colour and texture processing principles for image 
analysis. This performs a scene segmentation based on colour and texture information. Drimbarean and Whelan [1] examined the contribution of colour information to the overall classification performance. They extended the grey level algorithms to colour images and found that the inclusion of colour increases the classification results without significantly complicating the feature extraction algorithms. Pietiekainen et al. 5] presented a colour texture classification based on separate processing of complementary colour and pattern information. From the classification results they concluded that colour and texture have complementary roles.

Though there exist many techniques for analysing colour and textures, processing of colour textured images is still a relatively undeveloped field and there does not yet exist a reliable model which can employ both colour and texture at the same time. There are few standard methods that are widely used to incorporate the chromatic information into texture analysis.

- Each colour band processed separately. This standard method extends the greyscale approaches such as Co-occurrence, Local Binary Pattern (LBP), Discrete Cosine Transform (DCT), etc., to the colour images and the result is obtained in each plane separately.

- Interaction between spectral bands. This approach uses the spatial interaction between different spectral bands.

- Use of individual colour band and cross-band information. This technique employs the information from both unichrome features and opponent features.

- Colour and texture information processed separately. In this approach the colour signal is divided into luminance and chrominance components and are processed separately. Grey level algorithms are applied to the intensity image and colour information from the chrominance plane is used as an additional information.

This paper focuses on the last approach, in which colour and texture information are processed separately. The proposed method uses both colour and texture features for colour texture segmentation. Research on human visual system supports the processing of luminance and chrominance components separately. LBP and the DCT developed by Ojala et al. [6] and $\mathrm{Ng}$ et al. 7] respectively are used as the feature extraction techniques to extract features from the intensity plane, followed by the extraction of colour features from the chrominance planes. An unsupervised texture segmentation method [8] is used to segment the image. A pixelwise classification is pursued to enhance the segmented result on the boundaries. This paper is organised as follows: Section 2 describes the different colour spaces. Section 3 details the colour texture segmentation method. Section 4 presents the experimental results and Section 5 concludes the paper.

\section{Colour Spaces}

A colour space is a model for representing colours in terms of intensity values. Colour model is the geometric representation of colours in a three dimensional 
space. RGB colour space is the fundamental colour space in image processing. This is an additive space based on tri-chromatic theory. Different colour spaces are better for different applications. Some colour spaces turn out to be application dependent. Hence, for an improved colour processing a more appropriate method is to utilise a different colour space, where the same information is represented in a way that corresponds better to the segmentation method. The various colour spaces in scientific use are HSI, HSV, YIQ, CIE-XYZ, CIE-LAB, CIE-LUV etc. The advantage of the colour texture model described in this paper is that it can be applied to any colour space, although we will focus on YIQ and HSI colour spaces for the purpose of this paper. YIQ is a linear and HSI is nonlinear transformation of RGB cube. HSI representation of colour is close to the method of colour description used by humans [9]. The YIQ and HSI systems separate colour information of an image from its intensity information. Grey level algorithms can be applied to the intensity plane and the texture information is extracted from the luminance plane i.e from Y plane in YIQ and I plane in HSI space. The colour information is extracted from the two chrominance planes.

\section{Colour Texture Segmentation}

This section describes the feature extraction techniques, and the method adopted for colour texture segmentation.

LBP is one of the feature extraction technique used which is invariant against any monotonic grey scale transformation and is computationally efficient. This technique is based on the two level version of the texture spectrum method, and it provides knowledge about the spatial structure of the local image texture. LBP's also provide robust pattern related information. Ojala et al. [6] carried out texture classification based on feature distributions of different texture measures and found that this method performed well when applied to Brodatz [10] textures. LBP is combined with the contrast of the texture which is the measure of local variations present in an image.

For comparison, we selected the DCT suggested by $\mathrm{Ng}$ et al. 7]. Randen and Husoy 11 found relatively good results using the DCT approach. Local linear properties can be extracted using well known transforms similar to DCT. Here a $3 \times 3 \mathrm{DCT}$ is used for texture feature extraction, and the one dimensional filter masks $h_{1}=[1,1,1], h_{2}=[1,0,-1], h_{3}=[1,-2,1]$ are used for the implementation. This yields 9 independent $3 \times 3$ DCT masks which generates a 9 -dimensional feature vector. The DCT is orthogonal and separable and is widely used in image coding applications.

Pure colour features such as mean, standard deviation, energy, entropy are computed as the distribution of image region. Each colour feature is computed from the chrominance planes and they are quantised to 8 bins for each colour plane. The distribution of LBP and contrast are used in conjunction with the distribution of the colour features for the colour texture description. On the other hand, the 9 DCT feature vectors are combined with the distribution of the quantised colour features for colour texture segmentation. A dissimilarity measure, 


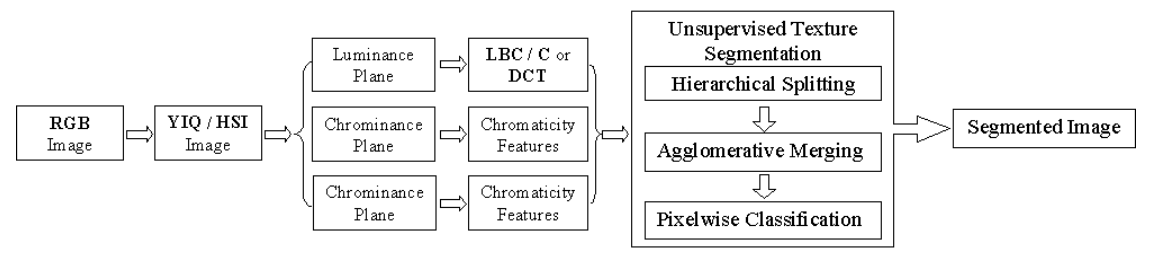

Fig. 1. An overview of the colour texture segmentation

G-Statistic [8], is used for colour texture discrimination. In this paper an unsupervised texture segmentation method developed by Ojala et al. [8] is used. In unsupervised texture segmentation, statistical analysis is performed on the entire distribution of vectors, and the aim is to recognise clusters in the distribution and assign the same label to them all. This method follows hierarchical splitting and agglomerative merging procedure. The hierarchical splitting divides the image into blocks of roughly uniform texture and agglomerative merging procedure merges the similar adjacent regions until a stopping rule is satisfied [8]. We proposed a novel data structure to implement the algorithm for unsupervised texture segmentation [12. The proposed algorithm implements the splitting using a quadtree structure and merging by mergegraph which generates a forest structure with each tree in the forest representing a merged area in the image. This is followed by a pixelwise classification to enhance the boundaries of the segmented image.

\section{Experimental Results}
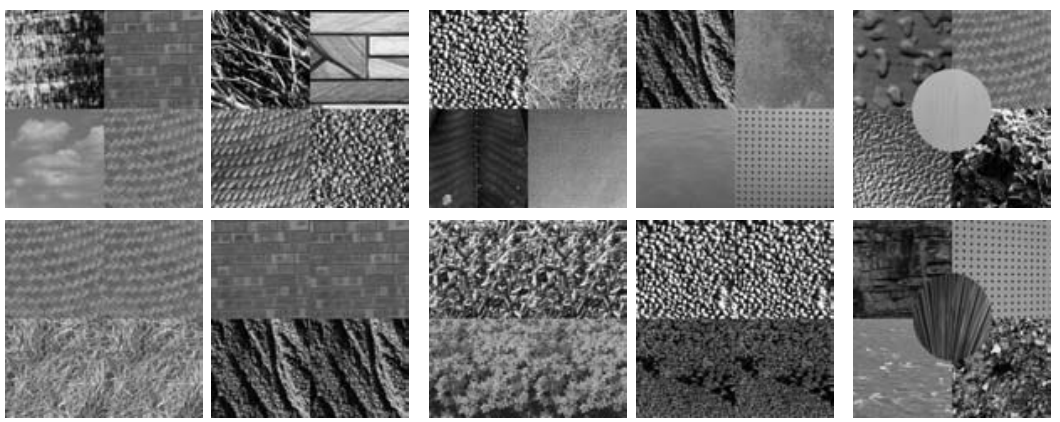

Fig. 2. Mosaics of colour texture images

The evaluation of the proposed method was performed on a set of 10 colour texture mosaic images obtained from VisTex [13] image database. The size of each image is $256 \times 256$, constructed using random selection of four images of size 
$128 \times 128$. The effectiveness of the proposed method was found by conducting various experiments. Ten VisTex images were processed using the segmentation algorithm with various parameter values.

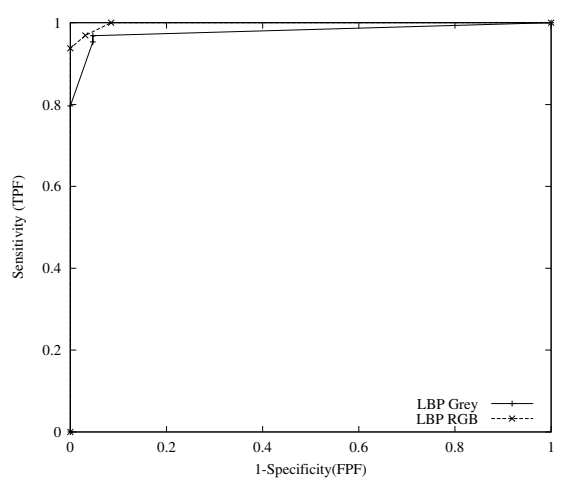

(a)

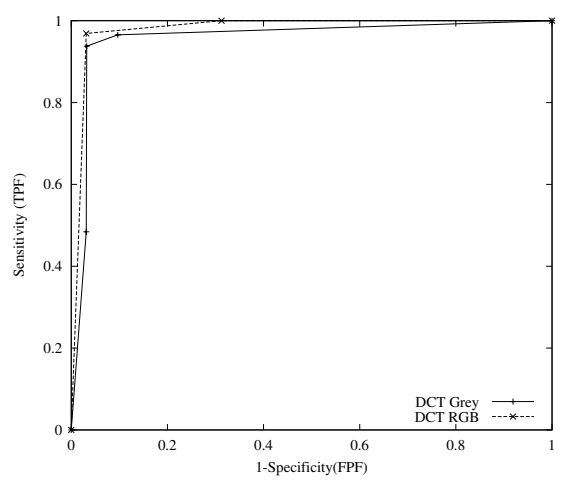

(b)

Fig. 3. (a) ROC curves for LBP approach in greyscale and RGB space (b) ROC curves for DCT approach in greyscale and RGB space

The first phase of the experiment demonstrates the performance of the grey level and colour texture images. As a quantitative analysis, the Receiver Operating Characteristic Curve (ROC) is drawn to determine the efficiency of the method. The segmentation results are evaluated using segmentation errors. The number of pixels classified in the segmented image and the number of pixels misclassified in the segmented image were considered for drawing the ROC curve. The ground truth of the image was obtained by manually drawing boundaries between different textured regions.

Table 1. Segmentation results (\% correctly segmented) for the first phase of the experiment

\begin{tabular}{|l|l|l|}
\hline Technique & Greyscale & RGB \\
\hline LBP & $89.6 \%$ & $94 \%$ \\
\hline DCT & $81.8 \%$ & $90.7 \%$ \\
\hline
\end{tabular}

The segmentation results in Table 1 illustrates that the inclusion of colour increases the performance of the segmentation. The columns correspond to average percentage of the segmentation results using LBP and DCT approach in greyscale and RGB space. The algorithm works excellently and the result is $100 \%$ for the images with square regions. Comparatively no order of ranking was mentioned for the approaches based on the segmentation results. Relating 
to the processing time, on an average both the approaches produced results in 1.28 seconds as they are computationally simple. The second phase of the exper-

Table 2. Segmentation results (\% correctly segmented) for the second phase of the experiment

\begin{tabular}{|l|l|l|l|l|l|}
\hline Technique & $\begin{array}{l}\text { Colour } \\
\text { Space }\end{array}$ & \multicolumn{4}{|c|}{ Chromaticity Features } \\
\cline { 3 - 6 } & & Mean & Stddev & Energy & Entropy \\
\hline \hline LBP & RGB & $94 \%$ & $92.1 \%$ & $77.3 \%$ & $75.1 \%$ \\
& YIQ & $93.7 \%$ & $91.3 \%$ & $97.2 \%$ & $91.4 \%$ \\
& HSI & $92.1 \%$ & $89.3 \%$ & $88.4 \%$ & $97.7 \%$ \\
\hline DCT & RGB & $76.4 \%$ & $90.7 \%$ & $82.6 \%$ & $87.2 \%$ \\
& YIQ & $91.3 \%$ & $75.5 \%$ & $81.1 \%$ & $98 \%$ \\
& HSI & $80.6 \%$ & $85.6 \%$ & $80.1 \%$ & $92.1 \%$ \\
\hline
\end{tabular}

iment was carried out to show that the chromaticity features play a vital role in colour texture segmentation. This phase allows a comparison of chromaticity features. Mean, standard deviation, energy and entropy are the features exploited. These are the standard features used by Haralick [14]. Different chromaticity features are extracted from the chrominance planes together with the LBP or DCT features from the intensity plane. Table 2 lists the segmentation results using different chromaticity features in different colour spaces. Experimental results indicates that the chromaticity entropy gave results with greater accuracy. The illustrated results are obtained before pixelwise classification. Application of the pixelwise classification enhances the overall segmentation result. This approach was also tested using natural images. Although ideal segmentation was not obtained the results are satisfactory.

\section{Conclusion}

This study, presented a model for colour texture segmentation. The model investigates the contribution of colour in the analysis of textures. The experiments performed demonstrate the use of colour in texture analysis. From the segmented results, the role of colour can be observed by way of segmentation within a uniform texture region. The addition of colour plays a significant part and the inclusion of colour improves the segmentation results.

\section{References}

1. Drimbarean, A., Whelan, P.F.:Experiments in colour texture analysis. Pattern Recognition Letters. Vol. 22. (2001) 1161-1167 
2. Panjwani, D.K., Healey, G.:Markov Random Field models for unsupervised segmentation of textured colour images. IEEE Transactions on Pattern analysis and Machine Intelligence. Vol. 17(10). (1995) 939-954

3. Jain, A.K., Healey, G.:A multiscale representation including opponent colour features for texture recognition. IEEE Trans on Image Processing. Vol. 7(1). (1998) 124-128

4. Paschos, G., Valavanis, K.P.:A color texture based visual monitoring system for automated surveillance. IEEE Trans of Systems, Man, and Cybernetics-Part C:Applications and Reviews. Vol. 29(1). (1999) 298-307

5. Pietikäinen, M., Mäenpää, T., Viertola, J.:Color texture classification with color histograms and local binary pattern. Proc.Texture2002. 2nd International Workshop on Texture Analysis and Synthesis. In Conjunction with ECCV2002. (2002)

6. Ojala, T., Pietikäinen, M., Harwood, D.:A comparative study of texture measures with classification based on feature distributions. Pattern Recognition. Vol. 29(1). (1996) $51-59$

7. Ng, I., Tan, T., Kittler, J.:On local linear transform and gabor filter representation of texture. Proc.Int.Conf.Pattern Recognition. (1992) 627-631

8. Ojala, T., Pietikäinen, M.:Unsupervised texture segmentation using feature distributions. Pattern Recognition. Vol. 32. (1999) 477-486

9. Whelan, P.F., Molloy, D.:Machine Vision Algorithms in Java:Techniques and Implementation. Springer (London). (2000)

10. Brodatz, P.:Texture-A photographic Album for Artists and Designers. Reinfold, NewYork. (1968)

11. Randen, T., Husoy, J.H.:Filtering for Texture Classification: A Comparative Study. IEEE Trans on Pattern Analysis and Machine Intelligence. Vol. 21(4). (1999) 291310

12. Padmapriya, N., Pradeep, P.P., Whelan, P.F.:A new data structure for the implementation of unsupervised texture segmentation. Proc. of The Irish Signals and Systems Conference. (2002) 109-113

13. VisTex., Colour Image Database: http://www-white.media.mit.edu/vismod/ imagery/ VisionTexture/vistex.html. (2000)

14. Haralick., R.M.:Statistical and structural approaches to texture. Proc. IEEE. Vol. 67. (1979) 786-804 